\title{
The case for noncompetitive cyclizative options during attempted expedient construction of the core ring system of CP-263,114
}

\author{
Nicolas Cunière and Leo A. Paquette* \\ Evans Chemical Laboratories, The Ohio State University, \\ Columbus, Ohio 43210, USA \\ E-mail: Paquette.1@osu.edu
}

Dedicated to Professor Gurnos Jones on the occasion of his $70^{\text {th }}$ birthday (received 24 Mar 00; accepted 20 Aug 00; published on the web 28 Aug 00)

\begin{abstract}
The C-6 alkylation of 2-cyclohexenone with methyl bromoacetate was followed by brominationdehydrobromination of the double bond. Palladium-catalyzed coupling of 8 to dimethyl (E)-2hexenedioate provided the key triester 4. All attempts to bring about intramolecular oxidative cyclization of the dienolate of 4 was found to result in preferred formation of the Dieckmann product 9 instead.
\end{abstract}

Keywords: Palladium-catalyzed coupling, CP-263,114, ester enolate oxidations, Dieckmann condensation

\section{Introduction}

Recently, a research team at Pfizer discovered two structurally remarkable new compounds currently known as CP-225,197 (1) and CP-263,114 (2). ${ }^{1}$ These substances were identified in a fermentation broth generated from a Texas juniper fungus that co-produces zaragozic acid A (squalestatin 1). ${ }^{2,3}$ The high medicinal promise of 1 and 2 derives from their impressive inhibitory activity against protein farnesyl transferase, these compounds exhibiting an $\mathrm{IC}_{50}$ in rat brain of $6-20 \mu \mathrm{M}$. This enzyme is widely recognized to be responsible for catalyzing the addition of farnesyl pyrophosphate to a cysteine residue at the carboxy terminus of protein $\mathrm{p} 21$, a product of the ras oncogene. Under normal circumstances, a carcinogenic condition develops because of a one-amino acid mutation of p21 whose effect is to leave the protein in a permanently active state. Cell division and growth subsequently proceed in an uncontrolled manner. Should the initial addition step be impeded, the expectation is that the carcinogenic process will not be turned on. 4 
In a different context, 1 is also active against squalene synthase in rat liver microsomes $\left(\mathrm{IC}_{50}\right.$ $43 \mu \mathrm{M})$, the enzyme that catalyzes the co-condensation of farnesyl pyrophosphate to presqualene pyrophosphate on the way to squalene. Since this process is central to cholesterol biosynthesis, this agent or analogues thereof are expected to be serviceable cholesterol-lowering drugs. ${ }^{5}$

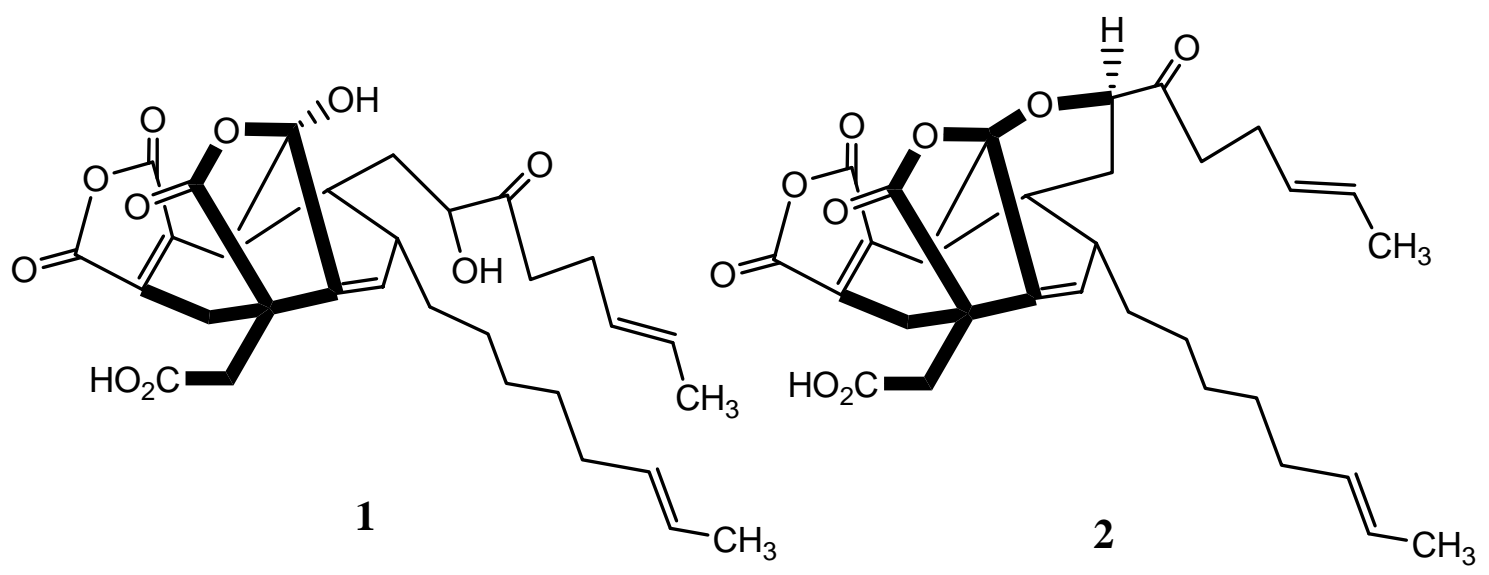

The intriguing structural features defined by these nonadrides have prompted the design of a divergent collection of routes to these systems, ${ }^{6-14}$ one of which has eventuated in a successful total synthesis. ${ }^{15}$ In connection with an attempt to achieve a short preparative route to the core ring system of 1 and 2, we have considered a pathway that would eventuate in the delivery of 3 in only five steps from 2-cyclohexenone (Scheme 1). However, the success of this potentially powerful strategy rests directly on our ability to effect proper intramolecular oxidative coupling of the dienolate of 4 without competitive operation of a Dieckmann cyclization. ${ }^{16}$ This brief report details the series of observations made in the context of this investigation.
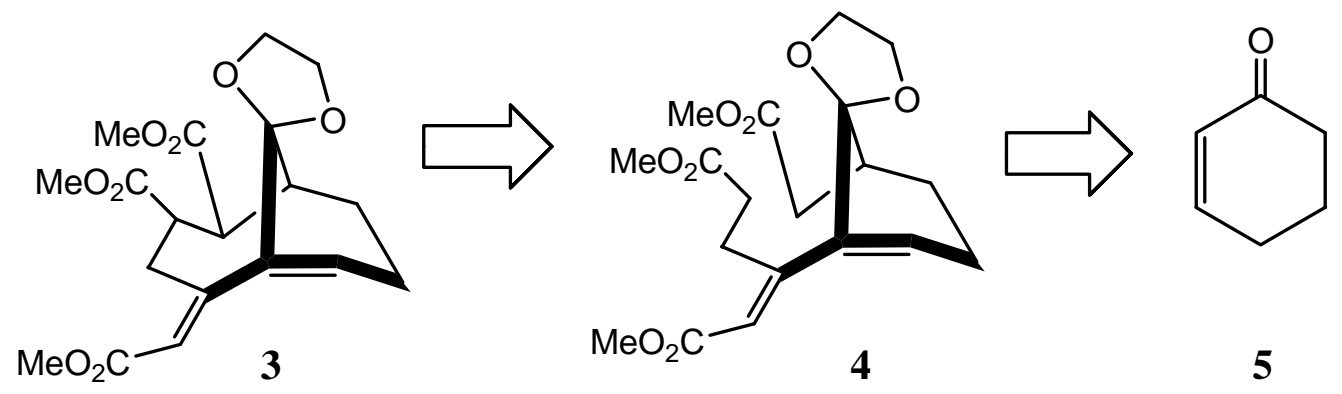

\section{Scheme 1}

The first transformation involved alkylation of the kinetic enolate of 5 with methyl bromoacetate $^{17}$ as shown in Scheme 2. Keto ester 6, thus obtained in $80 \%$ yield, proved entirely amenable to bromination-dehydrobromination as expected. 
<smiles>CC(=O)CC1CCC=C(Br)C1=O</smiles>

6

(85\%)

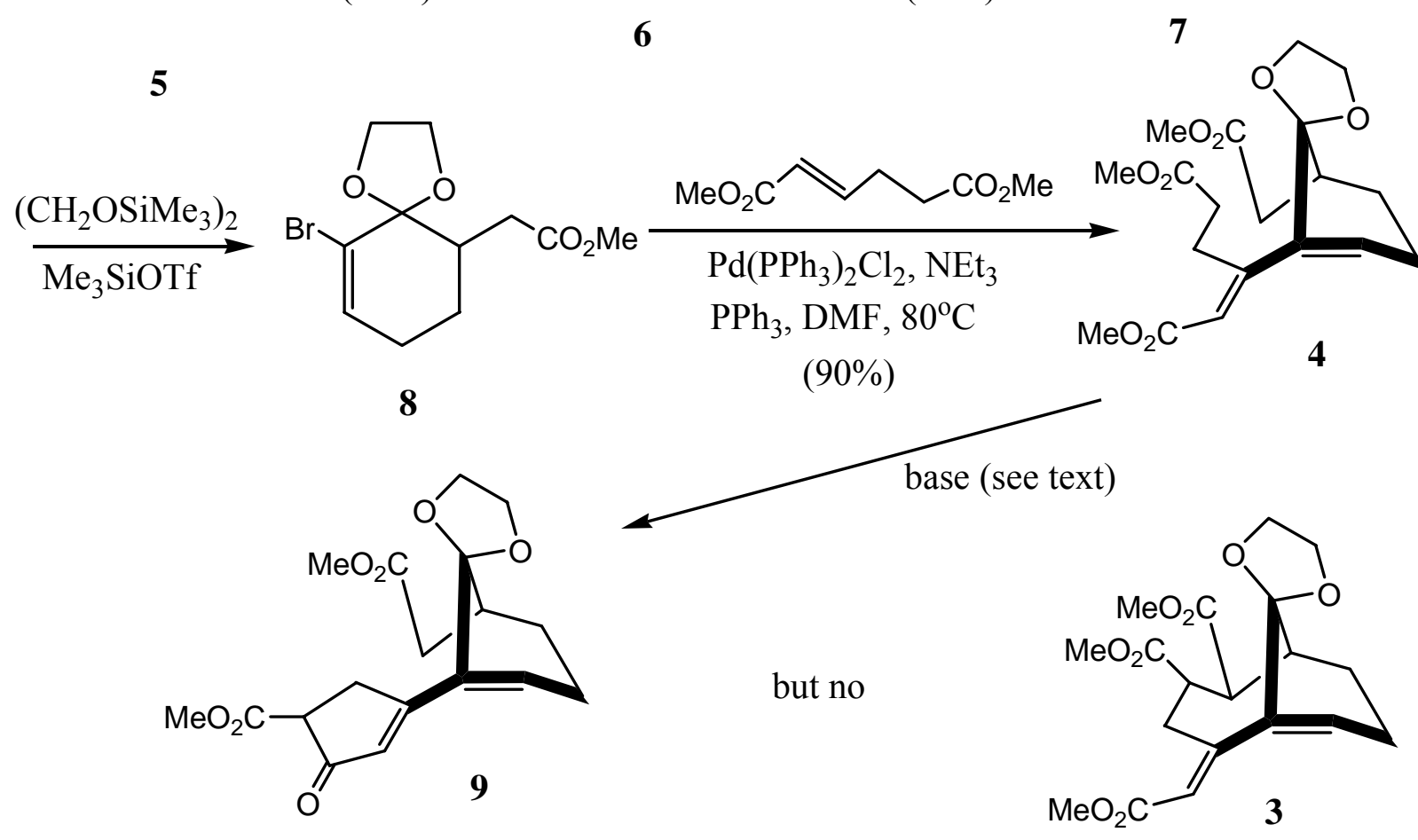

\section{Scheme 2}

The bromo enone 7 generated in this manner was conveniently responsive to acetalization under the Noyori conditions. ${ }^{18}$ Introduction of the diester-containing side chain was accomplished at this stage by stirring 8 with dimethyl $(E)$-hexenedioate under conventional Heck conditions. ${ }^{19}$ Application of this difunctional reagent, directly available from the palladiumcatalyzed dimerization of methyl acrylate, ${ }^{20}$ provided the key triester 4 .

The ability of cupric and ferric ions to bring about the oxidative coupling of ester enolates, recognized more than 20 years ago, ${ }^{21}$ has serviced several synthetic objectives in both its intermolecular $^{22-24}$ and intramolecular variants. ${ }^{25,26}$ Comparable success has been realized with elemental iodine as the oxidant. ${ }^{27,28}$ A variety of possibilities for effecting the conversion of 4 into 3 by these methods failed to achieve the desired carbon-carbon bond formation (Table I).

In those examples where consumption of starting material was evident, keto diester 9 could be isolated in yields up to $60 \%$. Alternative recourse to copper(II) bromide ${ }^{22}$ or silver(I) oxide (DMSO, $80{ }^{\circ} \mathrm{C}, 8$ days) $)^{29,30}$ fared no better. When 4 failed to respond to the combined action of potassium tert-butoxide and iodine, ${ }^{28}$ the decision was made to determine the readiness with which 4 entered into the Dieckmann condensation. 
Table 1. Repres entative Oxidation involving

\begin{tabular}{|c|c|c|c|c|}
\hline base & solwert & oxidant & temp, $\mathrm{PC}$ & product composition \\
\hline $\begin{array}{c}\text { LD出 } \\
\text { (3 equiv) }\end{array}$ & $\begin{array}{c}\text { THF-DMF } \\
(9: 1)\end{array}$ & $\mathrm{CuCl}_{2}$ (neat) & $-4 \square \rightarrow 0$ & $4(6 \square \%) ; 9(4 \square \%)$ \\
\hline $\begin{array}{c}\text { LDA } \\
\text { (3 equiv) }\end{array}$ & THF & $\mathrm{CuCl}_{2}(\mathrm{in} \mathrm{DM} / \mathrm{F})$ & $-4 \square \rightarrow \mathrm{rt}$ & $4(40 \%) ; 9(6 \square \%)$ \\
\hline $\begin{array}{l}\text { LiHMDS } \\
\text { (B equiv) }\end{array}$ & $\begin{array}{c}\text { THF-TMEDA } \\
(9: 1)\end{array}$ & $\mathrm{I}_{2}$ & $-7 \mathrm{~B} \rightarrow \mathrm{rt}$ & $4(50 \%) ; 9(50 \%)$ \\
\hline $\begin{array}{l}\text { LiTMP } \\
\text { ( equiv) }\end{array}$ & $\begin{array}{c}\text { THF-TMEDA } \\
(9: 1)\end{array}$ & $\mathrm{CuCl}_{2}(\mathrm{neat})$ & $-78 \rightarrow 0$ & $9(60 \%)$ \\
\hline
\end{tabular}

a In all four experiments, 4 and 9 were separated chromatographically

and identified by spectroscopic characterization.

Quite remarkably, treatment of 4 with 2.2 equiv of lithium 2,2,6,6-tetramethylpiperide (LiTMP) in THF at $-78{ }^{\circ} \mathrm{C}$ for 30 min furnished 9 in $70 \%$ yield.

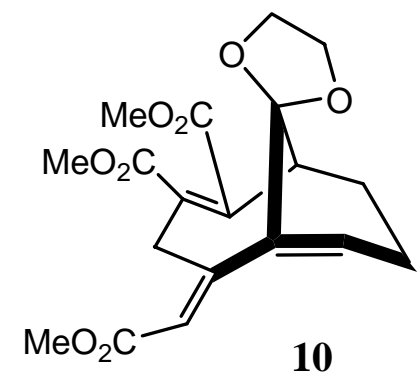

Quite evidently, the rate of five-ring closure associated with the Dieckmann pathway cannot be overridden by the kinetics associated with formation of the larger ring resident in 3 . Beyond that, the conversion of 4 into 9 does not require dienolate generation. In addition, 9 does not find it possible to return to 4 since deprotonation of the acidic hydrogen positioned in the 1,3dicarbonyl subunit guarantees irreversibility. Had 3 been produced, we had every expectation that chemoselective oxidation to 10 could have been accomplished by the Wilkening-Mundy protocol. ${ }^{31}$ 


\section{Experimental Section}

General Procedures. The column chromatographic separations were performed with Woelm silica gel (230-400 mesh). Solvents were reagent grade and in most cases dried prior to use. The purity of all compounds was shown to be $>95 \%$ by TLC and high field ${ }^{1} \mathrm{H}$ and ${ }^{13} \mathrm{C}$ NMR. The high-resolution and fast-atom-bombardment spectra were recorded at The Ohio State University Campus Chemical Instrumentation Center. Elemental analyses were performed at Atlantic Microlab, Inc., Norcross, GA.

Methyl 2-oxo-3-cyclohexene-1-acetate (6). To a solution of diisopropylamine (16 $\mathrm{mL}$, $0.11 \mathrm{~mol})$ in dry THF $(150 \mathrm{~mL})$ cooled to $-78^{\circ} \mathrm{C}$ was added $n$-buytllithium $(97 \mathrm{~mL}$ of $1.17 \mathrm{M}$ in hexanes, $0.11 \mathrm{~mol})$. After $15 \mathrm{~min}$, a solution of $5(10.0 \mathrm{~g}, 0.104 \mathrm{~mol})$ in dry THF $(50 \mathrm{~mL})$ was introduced dropwise over a period of $15 \mathrm{~min}$. After $3 \mathrm{~h}$ of stirring at $-78{ }^{\circ} \mathrm{C}$, the reaction mixture was quenched with saturated $\mathrm{NH}_{4} \mathrm{Cl}$ solution, the separated aqueous layer was extracted with ether, and the combined organic phases were washed with brine, dried and concentrated. The residue was distilled and further purified by flash chromatography on silica gel (elution with 1:1 hexanes/ether) to give 6 as a colorless oil ( $14 \mathrm{~g}, 80 \%$ ); bp $90{ }^{\circ} \mathrm{C}$ at 0.05 Torr; IR (neat, $\mathrm{cm}^{-1}$ ) 1735,$1681 ;{ }^{1} \mathrm{H}$ NMR $\left(300 \mathrm{MHz}, \mathrm{CDCl}_{3}\right) \delta$ 6.98-6.92 (m, 1H), 6.03-5.99 (m, 1H), $3.69(\mathrm{~s}, 3 \mathrm{H})$, 2.93-2.81 (m, 2H), 2.48-2.39 (m, 2H), 2.32-2.23 (m, 1H), 2.15-2.09 (m, 1H), 1.88-1.78 (m, 1H); ${ }^{13} \mathrm{C} \mathrm{NMR}\left(75 \mathrm{MHz}, \mathrm{CDCl}_{3}\right) \delta 199.3,172.9,149.8,129.2,51.6,43.6,34.2,28.6,25.9$; HRMS (EI) $\mathrm{m} / \mathrm{z}\left[\mathrm{M}^{+}\right]$calcd for $\mathrm{C}_{9} \mathrm{H}_{12} \mathrm{O}_{3} 168.0786$, obsd 168.0774.

Methyl 3-bromo-2-oxo-3-cyclohexene-1-acetate (7). To a solution of 6 (2.60 g, $0.015 \mathrm{~mol})$ in $\mathrm{CH}_{2} \mathrm{Cl}_{2}(30 \mathrm{~mL})$ at $-10{ }^{\circ} \mathrm{C}$ was added dropwise a solution of bromine $(0.78 \mathrm{~mL}, 1.05$ equiv $)$ in the same solvent $(30 \mathrm{~mL})$. After $30 \mathrm{~min}$, triethylamine $(2.59 \mathrm{~mL}, 1.2$ equiv) was introduced during 5 min, and the reaction mixture was stirred at $\mathrm{rt}$ for $3 \mathrm{~h}$ and quenched with saturated $\mathrm{NaHCO}_{3}$ solution. The organic phase was washed with water and brine, dried, and concentrated. Flash chromatography of the residue on silica gel (elution with $2: 1$ hexanes/ether) afforded $3.25 \mathrm{~g}$ $(85 \%)$ of 7 as an acid-sensitive colorless oil; IR (neat, $\left.\mathrm{cm}^{-1}\right) 1735,1687 ;{ }^{1} \mathrm{H} \mathrm{NMR}(300 \mathrm{MHz}$, $\left.\mathrm{C}_{6} \mathrm{D}_{6}\right) \delta 6.61-6.57(\mathrm{~m}, 1 \mathrm{H}), 3.38(\mathrm{~s}, 3 \mathrm{H}), 2.76(\mathrm{dd}, J=16.5,5.5 \mathrm{~Hz}, 1 \mathrm{H}), 2.60-2.50(\mathrm{~m}, 1 \mathrm{H}), 2.06$ $(\mathrm{dd}, J=16.5,6.9 \mathrm{~Hz}, 1 \mathrm{H}), 1.62-1.37(\mathrm{~m}, 3 \mathrm{H}), 1.28-1.14(\mathrm{~m}, 1 \mathrm{H}) ;{ }^{13} \mathrm{C} \mathrm{NMR}\left(75 \mathrm{MHz}, \mathrm{C}_{6} \mathrm{D}_{6}\right) \delta$ 190.7, 171.6, 149.9, 122.9, 50.9, 44.0, 34.5, 27.9, 27.4; HRMS(EI) $\mathrm{m} / \mathrm{z}\left[\mathrm{M}^{+}\right]$calcd 247.9891, obsd 247.9882. Anal. Calcd for $\mathrm{C}_{9} \mathrm{H}_{11} \mathrm{BrO}_{3}$ : C, 43.72; H, 4.48. Found: C, 43.69; H, 4.46.

Methyl 10-bromo-1,4-dioxaspiro[4.5]dec-9-ene-6-acetate (8). To a solution of trimethylsilyl triflate $(0.33 \mathrm{~mL}, 30 \mathrm{~mol} \%)$ in $\mathrm{CH}_{2} \mathrm{Cl}_{2}$ at $-78{ }^{\circ} \mathrm{C}$ was added 1,2-bis(trimethylsiloxy)ethane $(2.23 \mathrm{~mL}, 1.5$ equiv) and $7(1.50 \mathrm{~g}, 6.07 \mathrm{mmol})$. The reaction mixture was left to stand for 5 days at $\mathrm{rt}$, quenched with pyridine $(3 \mathrm{~mL})$, and diluted with ether and water. After the separated aqueous layer had been extracted with ether, the combined organic phases were washed with saturated $\mathrm{NaHCO}_{3}$ solution and brine, dried, and concentrated. Flash chromatography of the residue on silica gel (elution with $4: 1$ hexanes/ether) furnished $1.5 \mathrm{~g}(85 \%, 100 \%$ based on recovered 7) of 8 as a colorless oil; IR $\left(\mathrm{CH}_{2} \mathrm{Cl}_{2}, \mathrm{~cm}^{-1}\right) 1731 ;{ }^{1} \mathrm{H} \mathrm{NMR}\left(300 \mathrm{MHz}, \mathrm{C}_{6} \mathrm{D}_{6}\right) \delta 5.96-$ 
$5.94(\mathrm{~m}, 1 \mathrm{H}), 4.06-4.01(\mathrm{~m}, 1 \mathrm{H}), 3.99-3.92(\mathrm{~m}, 1 \mathrm{H}), 3.62-3.60(\mathrm{~m}, 2 \mathrm{H}), 3.42(\mathrm{~m}, 3 \mathrm{H}) 2.67-2.56$ $(\mathrm{m}, 2 \mathrm{H}), 2.17-2.09(\mathrm{~m}, 1 \mathrm{H}), 1.79-1.71(\mathrm{~m}, 1 \mathrm{H}), 1.63-1.57(\mathrm{~m}, 3 \mathrm{H}) ;{ }^{13} \mathrm{C} \mathrm{NMR}\left(75 \mathrm{MHz}, \mathrm{C}_{6} \mathrm{D}_{6}\right) \delta$ 172.3, 134.3, 125.0, 107.5, 66.6, 66.4, 50.8, 41.8, 33.9, 25.6, 25.1; HRMS (EI) m/z [M ${ }^{+}$calcd 292.0130, obsd 292.0098. Anal. Calcd for $\mathrm{C}_{11} \mathrm{H}_{15} \mathrm{BrO}_{4}$ : C, 45.35; H, 5.19. Found: C, 45.75; H, 5.24 .

(E)-3-[10-(Carboxymethyl)-1,4-dioxaspiro[4.5]dec-6-en-6-yl]-2-hexenedioic acid, trimethyl ester (4). A mixture of 8 (123 mg, $0.422 \mathrm{mmol})$, dimethyl (E)-2-hexenedioate $(0.17 \mathrm{~mL}, 2.5$ equiv), triethylamine $(0.11 \mathrm{~mL}, 2$ equiv), dichloropalladium bis(triphenylphosphine) (3 $\mathrm{mg}$, $1 \mathrm{~mol} \%)$, and triphenylphosphine $(6.6 \mathrm{mg}, 6 \mathrm{~mol} \%)$ in DMF $(0.1 \mathrm{~mL})$ was heated at $80{ }^{\circ} \mathrm{C}$ for 5 days, cooled, poured into water, and shaken with ether. The organic phase was washed with saturated $\mathrm{NaHCO}_{3}$ solution and brine, dried and concentrated. Flash chromatography of the residue on silica gel (elution with 1:1 ether/hexanes) gave $40 \mathrm{mg}(25 \%, 90 \%$ based on recovered 8) of 4 as a colorless oil; IR $\left(\mathrm{CH}_{2} \mathrm{Cl}_{2}, \mathrm{~cm}^{-1}\right) 1731,1729,1713 ;{ }^{1} \mathrm{H}$ NMR $\left(300 \mathrm{MHz}, \mathrm{C}_{6} \mathrm{D}_{6}\right) \delta 6.08$ $(\mathrm{s}, 1 \mathrm{H}), 5.49(\mathrm{t}, J=3.6 \mathrm{~Hz}, 1 \mathrm{H}), 3.77-3.69(\mathrm{~m}, 1 \mathrm{H}), 3.61-3.36(\mathrm{~m}, 4 \mathrm{H}), 3.44(\mathrm{~s}, 3 \mathrm{H}), 3.42(\mathrm{~s}, 3 \mathrm{H})$, 3.39 (s, 3H), 3.31-3.24 (m, 1H), 2.70-2.63 (m, 2H), 2.60-2.50 (m, 2H), 2.11-2.05 (m, 1H), 1.98$1.92(\mathrm{~m}, 1 \mathrm{H}), 1.75-1.70(\mathrm{~m}, 2 \mathrm{H}), 1.61-1.57(\mathrm{~m}, 1 \mathrm{H}) ;{ }^{13} \mathrm{C} \mathrm{NMR}\left(75 \mathrm{MHz}, \mathrm{C}_{6} \mathrm{D}_{6}\right) \delta 172.6(2 \mathrm{C})$, 166.1, 159.3, 139.2, 131.7, 119.3, 103.3, 65.3, 64.6, 50.8, 50.7, 50.3, 38.6, 33.2, 32.3, 27.7, 24.3, 22.2; HRMS (EI) $\mathrm{m} / \mathrm{z}\left[\mathrm{M}^{+}\right]$calcd for $\mathrm{C}_{19} \mathrm{H}_{26} \mathrm{O}_{8} 382.1627$, obsd 382.1593 .

10-(4-Carboxy-3-oxo-1-cyclopenten-1-yl)-1,4-dioxaspiro[4.5]dec-9-ene-6-acetic acid, dimethyl ester (9). A cold $\left(-78{ }^{\circ} \mathrm{C}\right)$, magnetically stirred solution of lithium 2,2,6,6tetramethylpiperidide ( 2.2 equiv) in dry THF $(1.2 \mathrm{~mL})$ was treated dropwise with a solution of 4 $(8 \mathrm{mg}, 0.02 \mathrm{mmol})$ in THF $(0.5 \mathrm{~mL})$. After $30 \mathrm{~min}$ at $-78{ }^{\circ} \mathrm{C}$, saturated $\mathrm{NaHCO}_{3}$ solution was introduced, to be followed by ether once warming to $\mathrm{rt}$ had occurred. The separated aqueous layer was extracted into ether, and the combined organic phases were washed with saturated $\mathrm{NaHCO}_{3}$ solution and brine, dried, and freed of solvent. Purification of the residue by flash chromatography on silica gel (elution with 1:1 ether/hexanes) afforded $9(5.2 \mathrm{mg}, 70 \%)$ as a 1:1 mixture of diastereomers; IR $\left(\mathrm{CH}_{2} \mathrm{Cl}_{2}, \mathrm{~cm}^{-1}\right) 1742,1699,1264 ;{ }^{1} \mathrm{H}$ NMR $\left(300 \mathrm{MHz}, \mathrm{CDCl}_{3}\right) \delta$ 6.58-6.55 (m, 1H), 6.20 (brs, 1H), 4.12-4.04 (m, 5H), $3.81(\mathrm{~s}, 3 \mathrm{H}), 3.75-3.66(\mathrm{~m}, 1 \mathrm{H}), 3.69(\mathrm{~s}$, $3 \mathrm{H}), 3.20$ (dd, $J=18.2,1.2 \mathrm{~Hz}, 1 \mathrm{H})(\mathrm{a}), 3.12$ (dd, $J=18.2,1.0 \mathrm{~Hz}, 1 \mathrm{H})(\mathrm{b}), 2.61-2.51$ (m, 2H), 2.35-2.30 (m, 2H), 2.22-2.16 (m, 1H), 2.04-2.01 (m, 1H), 1.78-1.72 (m, 1H); ${ }^{13} \mathrm{C} \mathrm{NMR}(75 \mathrm{MHz}$, $\left.\mathrm{CDCl}_{3}\right) \delta 197.6(\mathrm{a}), 197.4(\mathrm{~b}), 173.2(\mathrm{a}+\mathrm{b}), 168.6(\mathrm{a}), 168.4(\mathrm{~b}), 167.5(\mathrm{a}+\mathrm{b}) 140.7$ (a), 140.6 (b), $133.0(a+b), 124.9(a), 124.8(b), 107.7(a+b), 64.9(a+b), 64.8(a+b), 53.9(a+b), 51.7$ (2a), 51.5 (2b), 46.3 (a), 46.2 (b), 37.0 (a), 36.9 (b), 33.4 (a) 33.1 (b), 23.4 (a + b), 22.8 (a), 22.5 (b); FAB MS m/z $\left[\mathrm{M}^{+}+\mathrm{H}\right]$ calcd for $\mathrm{C}_{18} \mathrm{H}_{23} \mathrm{O}_{7} 351.15$, obsd 351.25.

\section{References and Notes}

1. (a) Dabrah, T. T.; Kaneko, T.; Massefski, W., Jr.; Whipple, E. B. J. Am. Chem. Soc. 1997, 119, 1594. (b) Dabrah, T. T.; Harwood, H. J., Jr.; Huang, L. H.; Jankovich, N. D.; Kaneko, 
T.; Li, J.-C.; Lindsey, S.; Moshier, P. M.; Subashi, T. A.; Therrien, M.; Watts, P. C. J. Antibiot. 1997, 56, 1.

2. Bergstrom, J. D.; Kurtz, M. M.; Rew, D. J.; Amend, A. M.; Karkas, J. K.; Bostedor, R. G.; Bansal, V. S.; Dufresne, C.; VanMiddlesworth, F. L.; Hensens, O. D.; Leisch, J. M.; Zink, D. L.; Wilson, K. E.; Onishi, J.; Milligan, J. A.; Kurtz, M. B.; Bills, G.; Bartizal, K. F.; Rozdilsky, W. A.; Abruzzo, G. K.; Kaplan, L.; Omstead, M. N.; Jenkins, R. G.; Huang, L.; Meinz, M. S.; Quinn, L.; Burg, R. W.; Kong, Y. L.; Mochales, S.; Mojena, M.; Martin, I.; Pelaez, F.; Diez, M. T.; Alberts, A. W. Proc. Natl. Acad. Sci. U.S.A.1993, 90, 80.

3. Dawson, M. J.; Farthing, J. E.; Marshall, P. S.; Middleton, R. F.; O’Neill, M. J.; Shuttleworth, A.; Stylli, C.; Tait, R. M.; Taylor, P. M.; Wildman, H. G.; Buss, A. D.; Langley, D.; Hayes, M. V. J. Antibiot. 1992, 45, 639.

4. (a) Leonard D. M. J. Med. Chem. 1997, 40, 2971 (b) Buss, J. E.; Morsters, J. C. Chem. Biol. 1995, 2, 787

5. Biller, S. A.; Neuenschwander, K.; Ponpipom, M. M.; Poulter, C. D. Curr. Pharm. Design 1996, 2, 1 .

6. (a) Sgarbi, P. W. M.; Clive, D. L. J. Chem. Commun. 1997, 2157. (b) Clive, D. L. J.; Sun, S.; He, X.; Zhang, J.; Gagliardini, V. Tetrahedron Lett. 1999, 40, 4605. (c) Clive, D. L. J.; Zhang, J. Tetrahedron 1999, 55, 12059

7. Davies, H. L. M.; Calvo, R.; Ahmed, G. Tetrahedron Lett. 1997, 38, 1737.

8. Armstrong, A.; Critchley T. J.; Mortlock, A. A. Synlett 1998, 552.

9. Chen, C.; Layton, M. E.; Shair, M. D. J. Am. Chem. Soc. 1998, 120, 10784.

10. Waizumi, N.; Itoh, T.; Fukuyama, T. Tetrahedron Lett. 1998, 39, 6015.

11. Bio, M. M.; Leighton, J. L. J. Am. Chem. Soc. 1999, 121, 890.

12. Yoshimitsu, T.; Yanagiya, M.; Nagaoka, H. Tetrahedron Lett. 1999, 40, 5215.

13. Devaux, J.-F.; O’Neil, S. V.; Guillo, N.; Paquette, L. A. submitted for publication.

14. (a) Kwon, O.; Su, D.-S.; Meng, D.; Deng, W.; D’Amico, D. C.; Danishefsky, S. J. Angew. Chem., Int. Ed. 1998, 37, 1877. (b) Kwon, O.; Su, D.-S.; Meng, D.; Deng, W.; D’ Amico, D. C.; Danishefsky, S. J. Angew. Chem., Int. Ed. 1998, 37, 1880. (c) Meng, D.; Danishefsky, S. J. Angew. Chem., Int. Ed. 1999, 38, 1485. (d) Meng, D.; Tau, Q.; Danishefsky, S. J. Angew. Chem., Int. Ed.1999, 38, 3197.

15. (a) Nicolaou, K. C.; Harter, M. W.; Boulton, L.; Jandeleit, B. Angew. Chem., Int. Ed. 1997, 36, 1194. (b) Nicolaou, K. C.; Postema, M. H. D.; Miller, N. D.; Yang, G. Angew. Chem., Int. Ed. 1997, 36, 2821. (c) Nicolaou, K. C.; He, Y.; Fong, K. C.; Yoon, W. H.; Choi, H.-S.; Zhong, Y.-L.; Baran, P. S. Org. Lett. 1999, 1, 63. (d) Nicolaou, K. C.; Baran, P. S.; Jautelat, R.; He, Y.; Fong, K. C.; Choi, H.-S.; Yoon, W. H.; Zhong, Y.-L. Angew. Chem., Int. Ed. 1999, 38, 549. (e) Nicolaou, K. C.; Baran, P. S.; Zhong, Y.-L.; Choi, H.-S.; Yoon, W. H.; He, Y.; Fong, K. C. Angew. Chem., Int. Ed. 1999, 38, 1669. (f) Nicolaou, K. C.; Baran, P. S.; Zhong, Y.-L.; Fong, K. C.; He, Y.; Yoon, W. H.; Choi, H.-S. Angew. Chem., Int. Ed. 1999, 38, 1676.

16. Schaefer, J. P.; Bloomfield, J. J. Org. React. 1967, 15, 1. 
17. Podraza, K. F.; Bassfield, R. L. J. Org. Chem. 1989, 54, 5919.

18. Tsunoda, T.; Suzuki, M.; Noyori, R. Tetrahedron Lett. 1980, 21, 1357.

19. Dieck, H. A.; Heck, R. F. J. Org. Chem. 1975, 40, 1083.

20. Nugent, W. A.; Hobbs, F. W., Jr. Org. Synth., Coll. 1993; Vol. VIII p 112.

21. Ito, Y.; Konoike, T.; Saegusa, T. J. Am. Chem. Soc. 1975, 97, 2912.

22. Rathke, M. W.; Lindert, A. J. Am. Chem. Soc. 1971, 93, 4605.

23. Ito, Y.; Konoike, T.; Harada, T.; Saegusa, T. J. Am. Chem. Soc. 1977, 99, 1487.

24. Frazier, R. H., Jr.; Harlow, R. L. J. Org. Chem. 1980, 45, 5408.

25. Chung, S. K.; Dunn, L. B., Jr. J. Org. Chem. 1983, 48, 1125.

26. Babler, J. H.; Sarussi, S. J. J. Org. Chem. 1987, 52, 3462.

27. Brocksom, T. J.; Petragnani, N.; Rodrigues, R.; La Scala Teixeira, H. Synthesis, 1975, 396.

28. Mazzega, M.; Fabris, F.; Cossu, S.; De Lucchi, O.; Lucchini, V.; Valle, G. Tetrahedron 1999, 55, 4427.

29. West, K. F.; Moore, H. W. J. Org. Chem. 1984, 49, 2809.

30. Ito, Y.; Fujii, S.; Koroike, T.; Saegusa, T. Synth. Commun. 1976, 6, 429.

31. Wilkening, D.; Mundy, B. P. Synth. Commun. 1984, 14, 227 